\title{
Ligand recognizing motifs in plant LysM receptors are major determinants of specificity
}

$$
\text { Zoltan Bozsoki }{ }^{1, \uparrow} \text {, Kira Gysel }{ }^{1, \uparrow} \text {, Simon B. Hansen }{ }^{1, \uparrow} \text {, }
$$

Damiano Lironi ${ }^{1}$, Christina Krönauer ${ }^{1}$, Feng Feng ${ }^{2}$, Noor de Jong ${ }^{1}$, Maria Vinther ${ }^{1}$, Manoj Kamble ${ }^{1}$, Mikkel B. Thygesen ${ }^{3}$, Ebbe Engholm ${ }^{3}$, Christian Kofoed ${ }^{3,4}$, Sébastien Fort ${ }^{5}$, John T. Sullivan ${ }^{6}$, Clive W. Ronson ${ }^{6}$, Knud J. Jensen ${ }^{3}$, Mickaël Blaise ${ }^{1,7}$, Giles Oldroyd ${ }^{2}$, Jens Stougaard ${ }^{1}$, Kasper R. Andersen ${ }^{1 *}$ and Simona Radutoiu ${ }^{1 * *}$

\section{Affiliations:}

${ }^{1}$ Department of Molecular Biology and Genetics, Aarhus University, 8000 Aarhus C, Denmark

${ }^{2}$ Sainsbury Laboratory, University of Cambridge, Cambridge CB2 1LR, UK

${ }^{3}$ Department of Chemistry, University of Copenhagen, 1871 Frederiksberg, Denmark

${ }^{4}$ Current Address: Frick Chemistry Laboratory, Department of Chemistry, Princeton University, Princeton NJ 08544, USA.

15 SUniv. Grenoble Alpes, CNRS, CERMAV, 38000 Grenoble, France.

${ }^{6}$ Department of Microbiology and Immunology, University of Otago, Dunedin 9054, New Zealand.

${ }^{7}$ Current Address: Institut de Recherche en Infectiologie de Montpellier, UMR 9004-CNRS, University of Montpellier, Montpellier, France.

*Correspondence to: kra@mbg.au.dk and radutoiu@mbg.au.dk

$\dagger$ These authors contributed equally

$\ddagger$ Lead Contacts 


\begin{abstract}
Plants evolved LysM receptors to recognize and parse microbial elicitors and drive intracellular signaling to limit or facilitate microbial colonization. Here we investigate chitin and Nod factor receptors of Lotus japonicus initiating differential signaling: immunity or root nodule symbiosis. Two motifs in their LysM1 domains determine specific recognition of ligands and

5 discriminate between their in planta functions. These motifs define the ligand binding site and comprise the most structurally divergent regions in cognate Nod factor receptors. An adjacent motif modulates the specificity for Nod factor recognition and determines the selection of compatible rhizobial symbionts in legumes. Finally, we identified how binding specificities in LysM receptors can be altered to facilitate Nod factor recognition and signaling from a chitin 10 receptor, advancing the prospects of engineering rhizobial symbiosis into non-legumes.
\end{abstract}

One Sentence Summary: Structure-guided engineering of specificities into immune and symbiosis LysM receptors 


\section{Main Text}

Glycan elicitors produced by bacteria and fungi are specifically recognized in plants and trigger the establishment of mutualistic or pathogenic associations (1-3). Receptors with extracellular LysM domains and intracellular kinases or pseudo-kinases present in all land plants often act in complexes (4-7) to perceive glycans and initiate specific signaling (-13). Phylogenetic studies revealed receptor relatedness, but remained short in providing indications of receptor function or ligand selectivity (14-16). Chitin hexamers (CO6) or larger oligomers released by fungi (17) are immunogenic to all land plants (18-21), but a common principle of chitin perception and signaling is lacking $(\underline{5}, \underline{6}, \underline{22})$. In legumes, $\operatorname{LjCERK6/MtCERK1}$ and $L j \mathrm{LYS} 13 / L j \mathrm{LYS} 14 / M t \mathrm{LYR} 4$ are required for chitin immunity, and both receptors of Medicago truncatula bind $\mathrm{CO} 8(6,21)$. Moreover, CO4-8 activated components of the pathway $(6,23)$ induced by lipochitooligosaccharides (LCOs) produced by symbionts, and CERK receptors were found important for symbiosis with arbuscular mycorrhiza (AM) fungi, suggesting a role for chitin and/or chitin perception in both immunity and AM symbiosis $(\underline{6}, \underline{24})$. These variations

15 indicate, that despite of its early emergence (25), the mechanism for chitin perception and signaling appears to have diversified over the course of plant evolution (22).

Symbiotic nitrogen-fixing bacteria produce structurally well-defined LCOs (Nod factors) that are recognized by legumes with high sensitivity $\left(10^{-12} \mathrm{M}\right)(\underline{26})$. This interaction is fully dependent on binding of distinctly decorated Nod factors to LysM receptors that control nodule organogenesis,

20 intracellular infection and nitrogen-fixation $(1, \underline{27-29})$. In pea, soybean and model legumes, a receptor with an active kinase (NFR1/LYK3 in Lotus/Medicago) and one with a pseudokinase (NFR5/NFP in Lotus/Medicago) are required for Nod factor signaling, and both NFR1 and NFR5 were found able to bind Nod factors $(\underline{30})$. Perception of Nod factors or chitin, and activation of a 
downstream signaling leading to root nodulation or immunity, operate in parallel in legume epidermal root cells $(\underline{21}, \underline{31})$.

Here we found that receptors recognizing Nod factors and chitin have a very similar structure but contain two diverging motifs in the LysM1 that are necessary for discriminating between immunity and symbiotic functions which can be further modulated by interactions at intracellular domains. These motifs define the ligand binding sites, and in Nod factor receptors an adjacent motif contributes to diversity in legume-rhizobia compatibility. We show how this knowledge can be used to alter the binding specificities in known LysM receptors, and engineer specific Nod factor recognition and signaling into a chitin receptor.

\section{LysM ectodomains are critical for nodulation and immunity.}

To understand how Nod factor or chitin perception and signaling is established by LysM receptors, we evaluated the contribution of corresponding domains (Fig. 1A, fig. S1) in Lotus japonicus (Lotus henceforth) NFR1 and CERK6 receptors. First, we investigated the contribution

15 of the ectodomain, transmembrane and juxtamembrane (TJ), and the kinase for signaling after $M$. loti inoculation or CO8 treatment. Intact NFR1 (1 in Fig. 1B, C) and all chimeras with the ectodomain of NFR1 $(2,3,4$ in Fig. 1B) induced nodule formation on $n f r 1-1$ (nfrl) mutants, but were not able to restore ROS production in cerk6-1 (cerk6) mutants after CO8 treatment $\mathbf{( 9 , 1 0 ,}$ 11, 12 in Fig. 1D). Chimeras enabled nodulation of $n f r l$ with different efficiencies. Chimera (3) 20 containing the kinase of CERK6 was less efficient than (1) or (2), while exchanging both TJ and kinase with CERK6 in (4) had a significant effect on nodulation. Only 2 out of the 33 plants expressing (4) formed nodules (Fig. 1B) indicating that combining elements present in the kinase and TJ regions of CERK6 had a negative impact on nodulation. All nodules were infected by $M$. 
loti bacteria (Fig. 1C and fig. S2), and we detected induction of the symbiotic marker pNin-GUS only in the nodulated roots (fig. S2). None of the chimeras containing CERK6 ectodomain triggered nodulation of $n f r l(\mathbf{5}, \mathbf{6}, \mathbf{7}, \mathbf{8})$. Analyses of the CO8-induced immune response found that CERK6 (13 in Fig. 1D) and chimera (14) enabled ROS production in cerk6 (Fig. 1D). In contrast, chimeras $(\mathbf{1 5 / 1 6})$ containing CERK6 ectodomain and the NFR1 kinase were unable to trigger ROS in cerk6. This indicates that NFR1 and CERK6 kinases differ in their capacity to activate immunity. Expression in tobacco leaf cells of YFP-tagged receptors with the same domain structures present in receptors $(\mathbf{4 / 1 2}),(\mathbf{5 / 1 3})$, or $(\mathbf{7 / 1 5})$ localized at the plasma membrane mirroring synthesis and expression observed for NFR1 (1/9) or CERK6 (5/13) (fig. S3).

10 However, we can not rule out effects on folding, stability or expression levels for the chimeric receptors found nonfunctional throughout our in planta assays. Previous studies based on overexpression of chimeric receptors between Lotus and Arabidopsis LysM proteins pinpointed the crucial role of NFR1 ectodomains in symbiosis $(\underline{32}, \underline{33})$. Our results based on native expression of chimeric receptors derived from paralogous proteins showed that ectodomains of

15 NFR1 and CERK6 contain major determinants for ligand perception and signaling specificity that can be further modulated by their intracellular regions. Similar modulation of specificity in the final signaling output by the intracellular domains has been observed with Leucine-rich repeat receptor kinases (34).

\section{Nod factor and chitin selectivity are determined by the LysM1 domain}

To determine which LysM domain in NFR1 and CERK6 harbors ligand specificity determinants, we tested functionality of chimeric receptors where combinations of the three LysM domains originating from the two receptors were coupled either to NFR1 or CERK6 TJ and kinases (Fig. 
2A). We found that recognition of $M$. loti and $\mathrm{CO} 8$ was dependent on the origin of the LysM1 domain. All chimeras $(\mathbf{1 7}, \mathbf{1 8}, \mathbf{1 9})$ containing the NFR1 LysM1 restored nodulation (Fig. 2B) and infection of $n f r 1$ (fig. S4), while chimeras $(\mathbf{2 0}, \mathbf{2 1}, \mathbf{2 2})$ with CERK6 LysM1 did not (Fig. $2 \mathrm{~B}$ and fig. S4). Reciprocal results were obtained for CO8-induced ROS in cerk6, where roots expressing $(\mathbf{2 8}, \mathbf{2 9}, \mathbf{3 0})$ with LysM1 of CERK6 produced ROS, while $(\mathbf{2 5}, \mathbf{2 6}, \mathbf{2 7})$ containing the LysM1 from NFR1 were nonfunctional in immunity (Fig. 2C). Results from expression of $(\mathbf{1 8} / \mathbf{1 9})$ revealed a lower efficiency of nodulation when compared to (1 or 17), indicating a negative impact of CERK6 LysM2 on nodulation. Besides this modest influence, the origin of LysM2 and LysM3 had no major impact on nodulation or ROS production. Previous structural studies of AtCERK1 ectodomain identified a chitin binding site in LysM2 (프). Our study from Lotus attributes functional specificity to LysM1. We therefore assayed which of the putative LysM1 or LysM2 binding sites could be perturbed without functional consequences for NFR1 or CERK6. The structure of CERK6 and the chitin-bound structure of AtCERK1 were used to identify conserved amino acids in LysM1 and LysM2 that when mutated to a bulky residue,

15 could disrupt the possible binding pocket (Fig. 2D) (35). Functional analyses of receptors with substitutions in LysM1 (NFR1-I78W and CERK6-V79W) (23/31) or LysM2 (NFR1-I140W and CERK6-I141W) (24/32) revealed that only mutations affecting the LysM1 impaired the ability of receptors to induce symbiosis or immunity (Fig. 2B, C, fig. S3 and S4). These results explain previous observations where mutants in LysM1 of pea Sym37 (므), and Medicago Lyk3 were

20 found defective in symbiosis ( $\underline{37})$. Together, these provide evidence for the major role of LysM1 domain in determining the selectivity for Nod factor and chitin perception.

\section{Two regions in LysM1 are required for specific signaling}


To dissect which elements in LysM1 are important for NFR1 and CERK6 functions, we identified four structural regions with substantial sequence differences (I to IV, Fig. 2E) and tested their requirement for Nod factor and $\mathrm{CO} 8$ recognition (Fig. 2F, G). We found that regions I and III can be swapped between NFR1 and CERK6 with no detrimental consequence on nodulation (33/35 in Fig. 2F) or ROS production (37/39 in Fig. 2G). In contrast, regions II and IV were both necessary for receptor functions in planta (34, 36, 38, $\mathbf{4 0}$ in Fig. 2F, G and fig. S4). Expression in tobacco revealed that CERK6 chimeras with regions II and IV from NFR1 are produced and localized at the plasma membrane (fig. S3), indicating that our structure-aided strategy for exchanging regions between paralogous receptors preserved the structure and stability of the proteins. Next, we investigated whether the corresponding regions in LysM1 of orthologous receptors NFR1 and LYK3 (Fig. 3A, B) are required for recognition of Nod factors varying in their decorations at the reducing and non-reducing ends (Fig. 3C). For this, we first investigated the capacity of LYK3 to complement $n f r 1$, and of NFR1 to complement lyk3 (Fig. 3D, E and fig. S1). The absence of nodulation in these assays (41 in Fig. 3D and 46 in Fig. 3E) 15 supports the role of these receptors for symbiont recognition $(\underline{38}, \underline{39})$. Moreover, in both legumes, the receptor ectodomains were found necessary for symbiotic signaling $(\mathbf{4 2}, \mathbf{4 3}$, in Fig. 3D and 48, 49 in Fig. 3E and fig. S5). Embedding regions II and IV of LYK3 into NFR1 (1) or into chimera (42) abolished their capacity to induce nodulation in $n f r l$ by M. loti (44/45 in Fig. 3D). Parallel experiments in Medicago where II and IV of NFR1 were embedded into LYK3 (47) or chimera (49) revealed a similar lack of nodulation in lyk3 by S. meliloti (50/51 in Fig. 3E and fig. S5). Together, these results from in planta experiments provide support for presence of molecular determinants for Nod factor signaling specificity in the LysM1 of NFR1 and LYK3. 
In parallel, we recombinantly expressed the ectodomains of NFR1, LYK3 and CERK6 in insect cells (fig. S6), and tested their capacity to bind M. loti and S. meliloti Nod factors using biolayer interferometry (BLI) (fig. S6). This revealed that LYK3 binds its cognate $S$. meliloti Nod factor with $\mathrm{K}_{d}=27.4 \pm 0.4 \mu \mathrm{M}$ and showed an approximate 6-fold reduced binding $\left(\mathrm{K}_{d}=173.2 \pm 0.9\right.$ $\mu \mathrm{M})$ to the non-cognate Nod factor from M. loti (Fig. 3F). By contrast and less expected, NFR1 binds both cognate and non-cognate Nod factors with similar affinity, $\mathrm{K}_{d}=38.7 \pm 0.4$ and $\mathrm{K}_{d}=$ $22.1 \pm 0.2 \mu \mathrm{M}$, respectively (Fig. 3G). CERK6, and likewise, NFR1 with regions II and IV from CERK6 (fig. S6), showed no binding to M. loti (Fig. 3H) or S. meliloti (fig. S6) Nod factor. Together, these results from in planta and in vitro binding assays demonstrate the crucial role of regions II and IV in this class of LysM receptors with active kinases for recognition of chitinous ligands.

\section{LYK3 reveals structural differences in the LysM1 binding site}

Our detailed investigations of ectodomain regions were aided by the available crystal structure of CERK6 (21). For the Nod factor receptors, no structural information was available, limiting the understanding of how these proteins distinguish different chitinous ligands at the molecular level. We crystallized the ectodomain of LYK3, and determined the structure at an atomic resolution of $1.5 \AA$ (Table S1). The structure revealed a classical fold of three LysM domains in a clover-leaf arrangement stabilized by three disulfide bridges (Fig. 4A). Comparison of LYK3

20 and CERK6 showed that the overall fold was conserved and the two structures align well with an RMSD (root mean square difference) of $0.5 \AA$ (181 $\mathrm{C} \alpha$ atoms aligned) (Fig. 4B). The main structural differences were observed in the LysM1 domain. In particular, region IV revealed a different conformation in LYK3 compared to CERK6 (Fig. 4C, D). Mapping both regions II and 
IV onto the LYK3 structure showed that these constitute the major part of a putative ligand binding site containing the functionally important residues L77 from SYM37 (므), and the NFR1-I78 in (23) (Fig. 2B, Fig. 4E). Together, these observations further support the conclusion that these regions of LysM1 define a ligand binding site within the NFR1-type receptor ectodomains.

\section{Contrasting motifs characterize chitin and Nod factor binding sites}

The identification of regions in LysM1, necessary and structurally positioned for the recognition of chitinous ligands, prompted us to investigate whether these represent general features in Nod

10 factor and chitin receptors from legume species. We reasoned that residues responsible for Nod factor recognition will be diverse between legumes recognizing variable and species-defined decorations of Nod factors. By contrast, the chitin receptors we hypothesized to be conserved in the corresponding regions giving the invariable structure of this ligand. Alignments and modelling of the entire ectodomains revealed a high surface conservation across the core LysM2 15 and LysM3 domains (fig. S7). Most differences between species were found to be present in LysM1 of NFR receptors (fig. S7A). Further dissection revealed that residues 40-46 and 75-81 (amino acid numbers in LYK3) embedded in regions II and IV, were the most variable parts in LysM1 of NFRs (Fig. 5A and fig. S7A, B). By contrast, the corresponding regions in CERKs were found to be highly conserved (Fig. 5B and fig. S7C, D). Superposition of a chitin oligomer 20 onto the structure of CERK6 LysM1 domain and prediction of the ligand interaction properties based on AtCERK1-chitin binding (35), identified six residues in each of region II (GSNLTY) and IV (KDSVQA) that are structurally positioned to enable contact with the chitin molecule 
(Fig. 5B). These residues are highly conserved among legume CERKs and could represent CObinding motifs (Fig. 5B).

\section{Engineering receptors for specific Nod factor recognition}

Identification of the two regions variable in NFRs (Fig. 5A, B) raised the question whether Nod factor selectivity can be reprogrammed by using these molecular fingerprints. To answer this, we modified the non-signaling receptors $(\mathbf{4 1} / \mathbf{4 3})$ containing LYK3 ectodomain (Fig. 3D) by exchanging regions II and IV with corresponding from NFR1 and tested for M. loti recognition. Parallel experiments were performed in Medicago lyk3 where regions II and IV of NFR1 in (46/48) (Fig. 3E) were exchanged with LYK3 residues. The engineered chimeras enabled nodulation of $n f r l$ (52/53 in Fig. 5C and fig. S5), but not of lyk3 (54/55 in Fig. 5D and fig. S5). Similar to NFR1-CERK6 chimeras $(\mathbf{2}, \mathbf{3}, \mathbf{4})$ we found that the efficiency of signaling from the engineered ectodomain is modulated by the cognate intracellular regions $(\mathbf{5 2} / \mathbf{5 3})$ possibly by fine-tuning interaction with species-defined downstream signaling components. This indicates

15 that symbiosis with M. loti in Lotus can be gained by engineering the LysM1 of LYK3, but a similar strategy does not suffice in Medicago. To locate additional elements that contribute to $S$. meliloti recognition, we inspected the 23 sequences from legume receptors (fig. S7), and observed that region III in NFR-type receptors (residue 54-65 in LYK3) also contained considerable variation among legumes (fig. S7A, B). This region is spatially close to the

20 proposed binding site (Fig. 5A). We explored the role of this region for recognition of S. meliloti by testing additional NFR1-LYK3 chimeras $(\mathbf{5 6}, \mathbf{5 7}, \mathbf{5 8})$ (Fig. 5D) and found that addition of LYK3 region III enabled S. meliloti recognition (Fig. 5D and fig. S5), indicating that regions close to the Nod factor binding site are important for engineering specificity into these receptors. 


\section{Reprogramming CERK6 receptor to recognize Nod factors and mediate nodulation}

Initiation of nodulation by Nod factor-producing rhizobia is restricted to leguminous plants and Parasponia species (느), while chitin recognition is ubiquitous among plants. Based on our results of engineering LYK3 and NFR1 (Fig. 5C, D), we asked whether recognition of M. loti Nod factor can be further extended by engineering CERK6. Systematic replacement of regions I, II, III and IV in receptor (21) (Fig. 2A) containing LysM1 domain of CERK6, with corresponding NFR1, was insufficient for engineering perception of $M$. loti Nod factor $(\mathbf{5 9}, \mathbf{6 0}$, 61, 62 in Fig. 5C and fig. S5), indicating that a more complex engineering approach is required.

10 However, a cooperative involvement of NFR1 regions II and IV was found when testing receptor (63) that enabled nodulation of $n f r l$ (Fig. 5C and fig. S5). The overall nodulation induced by (63) was lower compared to NFR1 (1), but the frequency of complementation was high (60 out of 63 transformed plants formed nodules), showing that (63) is an efficient Nod factor receptor. Embedding NFR1 regions II and IV in chimera (8) (Fig. 1A) with the entire

15 CERK6 ectodomain resulted in few nodules per plant, and low nodulation efficiency (35 out of 95 transformed plants, fig. S5) (64 in Fig. 5C), while similar engineering of the full-length CERK6 (5 in Fig. 1A) was not sufficient to allow nodulation in $n f r 1$ (65 in Fig. 5C, fig. S5). This gradual reduction in the efficiency of nodulation may occur as a result of the observed negative impact of CERK6 LysM2 (18 and 19 in Fig. 2B), and CERK6 TJ and kinase (4 in Fig. 1B) on

20 nodulation. To resolve if these findings from in planta were a result of changes in Nod factor binding properties of CERK6 (Fig. 3H), we tested the ectodomain of (64) for in vitro binding to M. loti Nod factor. Unlike CERK6, this chimeric ectodomain had the capacity to bind M. loti Nod Factor (Fig. 5E) with an apparent $\mathrm{K}_{d}=46.5 \mu \mathrm{M}$, similar to NFR1 ectodomain (Fig. 3G), 
demonstrating that regions II and IV from NFR1 are sufficient for engineering M. loti recognition in CERK6 ectodomain.

Here we investigated the molecular mechanism behind recognition of immunogenic and symbiotic chitin-based glycans by LysM receptors that have an active kinase. Comparative structural and functional studies resolved the role of regions II and IV in LysM1 for creating a structurally-defined binding pocket that discriminates between $\mathrm{CO} 8$ and Nod factor ligands, while additional layers of signaling ability are enabled by the intracellular regions (Fig. 1B, D and Fig. 4C, D). Two motifs with a high degree of conservation were identified in regions II and IV of legume CERKs (Fig. 5D), likely reflecting their ability to recognize and bind the structurally invariable chitin ligand (Fig. 5D and fig. S7C, D). By contrast, Nod factor receptors show sequence degeneration in corresponding motifs (Fig. 5C), reflecting the diversity in legume-rhizobia compatibility and Nod factor structures (Fig. 5C and fig. S7A, B). LYK3 and NFR1 vary in their signaling flexibility. Regions II and IV from NFR1 are sufficient to enable recognition of M. loti by LYK3 in Lotus (Fig. 5A), while regions II, III and IV from LYK3 are 15 necessary to enable efficient recognition of S. meliloti by NFR1 in Medicago (Fig. 5B). Region III is variable between legume species (Fig. 5C and fig. S7A, B), and we envision this could be required for establishing species-specific interactions with the ligand-bound or unbound coreceptor of the NFR5/NFP class. In summary, we demonstrate that LysM receptors with an active kinase have programable capacity for ligand perception enabling rational engineering of 20 selective signaling. Our findings therefore provide a solid basis for addressing future challenges such as engineering highly sensitive receptor complexes that could enable hosts outside of the nodulation clade to perceive symbiotic signals from nitrogen-fixing rhizobia. 


\section{References}

1. J. Denarie, J. Cullimore, Lipo-oligosaccharide nodulation factors: a minireview new class of signaling molecules mediating recognition and morphogenesis. Cell 74, 951-954 (1993).

2. K. Tsukada et al., Rice receptor for chitin oligosaccharide elicitor does not couple to heterotrimeric G-protein: Elicitor responses of suspension cultured rice cells from Daikoku dwarf (d1) mutants lacking a functional G-protein $\alpha$-subunit. Physiologia Plantarum 116, 373 $382(2002)$.

3. A. A. Gust et al., Bacteria-derived peptidoglycans constitute pathogen-associated molecular patterns triggering innate immunity in Arabidopsis. J Biol Chem 282, 32338-32348 (2007).

4. J. Wan et al., LYK4, a lysin motif receptor-like kinase, is important for chitin signaling and plant innate immunity in Arabidopsis. Plant Physiol 160, 396-406 (2012).

5. Y. Cao et al., The kinase LYK5 is a major chitin receptor in Arabidopsis and forms a chitin-induced complex with related kinase CERK1. Elife 3, (2014).

15 6. F. Feng et al., A combination of chitooligosaccharide and lipochitooligosaccharide recognition promotes arbuscular mycorrhizal associations in Medicago truncatula. Nat Commun 10, 5047 (2019).

7. E. B. Madsen et al., Autophosphorylation is essential for the in vivo function of the Lotus japonicus Nod factor receptor 1 and receptor-mediated signalling in cooperation with Nod factor receptor 5. Plant Journal 65, 404-417 (2011).

8. H. Kaku et al., Plant cells recognize chitin fragments for defense signaling through a plasma membrane receptor. Proc Natl Acad Sci U S A 103, 11086-11091 (2006).

9. Y. Kawaharada et al., Receptor-mediated exopolysaccharide perception controls bacterial infection. Nature 523, 308-312 (2015).

25 10. S. Radutoiu et al., Plant recognition of symbiotic bacteria requires two LysM receptorlike kinases. Nature 425, 585-592 (2003).

11. J. Wan et al., A LysM receptor-like kinase plays a critical role in chitin signaling and fungal resistance in Arabidopsis. Plant Cell 20, 471-481 (2008).

12. R. Willmann et al., Arabidopsis lysin-motif proteins LYM1 LYM3 CERK1 mediate bacterial peptidoglycan sensing and immunity to bacterial infection. Proc Natl Acad Sci U S A 108, 19824-19829 (2011).

13. E. B. Madsen et al., A receptor kinase gene of the LysM type is involved in legumeperception of rhizobial signals. Nature 425, 637 (2003).

14. L. Buendia, A. Girardin, T. Wang, L. Cottret, B. Lefebvre, LysM Receptor-Like Kinase and LysM Receptor-Like Protein Families: An Update on Phylogeny and Functional Characterization. Front Plant Sci 9, 1531 (2018).

15. X. C. Zhang, S. B. Cannon, G. Stacey, Evolutionary genomics of LysM genes in land plants. BMC Evol Biol 9, 183 (2009).

16. G. V. Lohmann et al., Evolution and regulation of the Lotus japonicus LysM receptor 40 gene family. Mol Plant Microbe Interact 23, 510-521 (2010).

17. A. Sanchez-Vallet, J. R. Mesters, B. P. Thomma, The battle for chitin recognition in plant-microbe interactions. FEMS Microbiol Rev 39, 171-183 (2015).

18. G. Felix, M. Regenass, T. Boller, Specific perception of subnanomolar concentrations of chitin fragments by tomato cells: induction of extracellular alkalinization, changes in protein phosphorylation, and establishment of a refractory state. The Plant Journal 4, 307-316 (1993). 
19. P. Vander, V. r. KM, A. Domard, N. Eddine El Gueddari, B. M. Moerschbacher, Comparison of the ability of partially N-acetylated chitosans and chitooligosaccharides to elicit resistance reactions in wheat leaves. Plant Physiol 118, 1353-1359 (1998).

20. B. Zhang, K. Ramonell, S. Somerville, G. Stacey, Characterization of Early, ChitinInduced Gene Expression in Arabidopsis. Molecular Plant-Microbe Interactions ${ }^{\circledR}$ 15, 963-970 (2002).

21. Z. Bozsoki et al., Receptor-mediated chitin perception in legume roots is functionally separable from Nod factor perception. P Natl Acad Sci USA 114, E8118-E8127 (2017).

22. T. Shinya et al., Functional characterization of CEBiP and CERK1 homologs in arabidopsis and rice reveals the presence of different chitin receptor systems in plants. Plant Cell Physiol 53, 1696-1706 (2012).

23. A. Genre et al., Short-chain chitin oligomers from arbuscular mycorrhizal fungi trigger nuclear $\mathrm{Ca} 2+$ spiking in Medicago truncatula roots and their production is enhanced by strigolactone. New Phytol 198, 190-202 (2013).

15 24. G. Carotenuto et al., The rice LysM receptor-like kinase OsCERK1 is required for the perception of short-chain chitin oligomers in arbuscular mycorrhizal signaling. New Phytol 214, 1440-1446 (2017).

25. S. Bressendorff et al., An Innate Immunity Pathway in the Moss Physcomitrella patens. Plant Cell 28, 1328-1342 (2016).

20 26. G. E. Oldroyd, R. M. Mitra, R. J. Wais, S. R. Long, Evidence for structurally specific negative feedback in the Nod factor signal transduction pathway. Plant J 28, 191-199 (2001).

27. D. W. Ehrhardt, R. Wais, S. R. Long, Calcium spiking in plant root hairs responding to Rhizobium nodulation signals. Cell 85, 673-681 (1996).

28. J. A. Downie, S. A. Walker, Plant responses to nodulation factors. Curr Opin Plant Biol 2, 483-489 (1999).

29. S. Radutoiu et al., LysM domains mediate lipochitin-oligosaccharide recognition and Nfr genes extend the symbiotic host range. EMBO J 26, 3923-3935 (2007).

30. A. Broghammer et al., Legume receptors perceive the rhizobial lipochitin oligosaccharide signal molecules by direct binding. Proc Natl Acad Sci U S A 109, 13859-13864 (2012).

31. Y. Kawaharada et al., Differential regulation of the Epr3 receptor coordinates membranerestricted rhizobial colonization of root nodule primordia. Nature Communications 8, (2017).

32. T. Nakagawa et al., From defense to symbiosis: limited alterations in the kinase domain of LysM receptor-like kinases are crucial for evolution of legume-Rhizobium symbiosis. Plant J 65, 169-180 (2011).

35 33. W. Wang, Z. P. Xie, C. Staehelin, Functional analysis of chimeric lysin motif domain receptors mediating Nod factor-induced defense signaling in Arabidopsis thaliana and chitininduced nodulation signaling in Lotus japonicus. Plant J 78, 56-69 (2014).

34. U. Hohmann et al., Constitutive activation of leucine-rich repeat receptor kinase signaling pathways by BAK1-interacting receptor-like kinase 3 chimera. bioRxiv,

$40 \quad$ 2020.2002.2018.954479 (2020).

35. T. Liu et al., Chitin-induced dimerization activates a plant immune receptor. Science 336, 1160-1164 (2012).

36. V. Zhukov et al., The Pea Sym37 Receptor Kinase Gene Controls Infection-Thread Initiation and Nodule Development. Molecular Plant-Microbe Interactions 21, 1600-1608 (2008). 
37. P. Smit et al., Medicago LYK3, an entry receptor in rhizobial nodulation factor signaling. Plant Physiol 145, 183-191 (2007).

38. P. Lerouge et al., Symbiotic host-specificity of Rhizobium meliloti is determined by a sulphated and acylated glucosamine oligosaccharide signal. Nature 344, 781-784 (1990).

39. P. Rodpothong et al., Nodulation gene mutants of Mesorhizobium loti R7A-nodZ and nolL mutants have host-specific phenotypes on Lotus spp. Mol Plant Microbe Interact 22, 15461554 (2009).

40. M. J. Trinick, Symbiosis between Rhizobium and Non-Legume, Trema-Aspera. Nature 244, 459-460 (1973).

10 41. Z. Bozsoki et al., Receptor-mediated chitin perception in legume roots is functionally separable from Nod factor perception. Proc Natl Acad Sci U S A 114, E8118-E8127 (2017).

42. S. J. Kelly et al., Conditional requirement for exopolysaccharide in the MesorhizobiumLotus symbiosis. Mol Plant Microbe Interact 26, 319-329 (2013).

43. S. Radutoiu, L. H. Madsen, E. B. Madsen, A. M. Nielsen, J. Stougaard, Agrobacterium rhizogenes pRi TL-DNA integration system: A gene vector for Lotus japonicus transformation. Lotus Japonicus Handbook (2005), pp. 285-287.

44. E. Weber, C. Engler, R. Gruetzner, S. Werner, S. Marillonnet, A modular cloning system for standardized assembly of multigene constructs. PLoS One 6, e16765 (2011).

45. J. Schindelin et al., Fiji: an open-source platform for biological-image analysis. Nat

20 Methods 9, 676-682 (2012).

46. K. Katoh, J. Rozewicki, K. D. Yamada, MAFFT online service: multiple sequence alignment, interactive sequence choice and visualization. Brief Bioinform 20, 1160-1166 (2019). 47. R. A. M. Villanueva, Z. J. Chen, ggplot2: Elegant Graphics for Data Analysis, 2nd edition. Meas-Interdiscip Res 17, 160-167 (2019).

25 48. T. R. c. team. (2019).

49. E. Murakami et al., Epidermal LysM receptor ensures robust symbiotic signalling in Lotus japonicus. Elife 7, (2018).

50. W. Kabsch, Integration, scaling, space-group assignment and post-refinement. Acta Crystallogr D 66, 133-144 (2010).

30 51. A. J. Mccoy et al., Phaser crystallographic software. J Appl Crystallogr 40, 658-674 (2007).

52. P. Emsley, B. Lohkamp, W. G. Scott, K. Cowtan, Features and development of Coot. Acta Crystallogr D 66, 486-501 (2010).

53. P. D. Adams et al., PHENIX: a comprehensive Python-based system for macromolecular structure solution. Acta Crystallographica Section D-Structural Biology 66, 213-221 (2010).

Acknowledgments: We thank J. Keller and P-M. Delaux for providing access to genomic sequences before publication, S. Thirup for input in the start of the project, F. Pedersen for plant care, L.H. Madsen, E. Soumpourou and M. Downes for assistance with gene cloning. Funding: This work was supported by the Bill and Melinda Gates Foundation and UK government's 
Department for International Development (DFID) through Engineering the Nitrogen Symbiosis for Africa (ENSA; OPP11772165) and the Danish National Research Foundation grant no. DNRF79. S.F. received technical support of ICMG (FR 2607) mass spectrometry and NMR platforms and partial financial support of Labex ARCANE and CBH-EUR-GS (ANR-17-EURE0003), Glyco@Alps (ANR-15-IDEX-02), and PolyNat Carnot Institut (ANR-16-CARN-002501). Author contributions: Z.B. gene cloning and in planta functional analyses; K.G., purification and affinity studies of LYK3 and NFR1 ectodomains, crystallization of LYK3; S.B.H., purification of NFR1, CERK6, CERK6-NFR1 ectodomains and affinity studies; D.L., C.K., F.F, M.K., gene cloning and in planta functional analyses; N.dJ., gene cloning and plant transformation, M.V., protein purification, M.B.T., E.E., C.K., biotin tagging of Nod factors and CO8, S.F., CO8 synthesis, J.T.S., purification of Nod factors, K.J.J., M.B., G.O., J.S., conceived experiments, K.R.A., and S.R., conceived experiments, analyzed data and coordinated studies. K.R.A. and S.R. wrote the manuscript with input from K.G., S.B.H., D.L., C.K., G.O, and J.S. Competing interests: Some findings in this manuscript are submitted in patent applications. Z.B., K.G., S.B.H., J.S., K.R.A., S.R. are inventors on patent application 62/718,186 submitted by Aarhus University that covers LysM receptors: Genetically altered LysM receptors with altered agonist specificity and affinity. S.R., K.R.A., D.L., C.K., J.S. are inventors on patent application 63/027,151 submitted by Aarhus University that covers LysM Receptor Motifs. Data and materials availability: All data is available in the main text or the supplementary materials.

20 Crystallographic coordinates and structure factors are available from the PDB under the accession code 6XWE (DOI: 10.2210/pdb6XWE/pdb). Material request: Gene constructs used in this study for functional and biochemical assays are available from S. R. and K. R. A. under a material agreement with Aarhus University, Denmark. 


\section{Supplementary Materials:}

Materials and Methods

Figures S1-S7

Tables S1

References (41-53)

\section{Figure legends:}

Fig. 1. Chimeric receptors identify domains important for chitin and Nod factor signaling (A) Schematics of NFR1 and CERK6. Boxes represent the three LysM domains (LysM1, LysM2 and LysM3) together constituting the ectodomain (EC), transmembrane + juxtamembrane domain (TJ) and the kinase domain (KD). Dotted lines and amino acids specify boundaries for chimera design. (B) Nodules formed on $n f r 1$ expressing indicated chimeras. (C) Phenotype of $n f r 1$ roots expressing chimeras $(\mathbf{1} / \mathbf{8})$, scale bar $=5 \mathrm{~mm}$. (D) ROS production observed for wild

15 type (WT) and cerk6 expressing indicated constructs. In (B) and (D) letters indicate significant differences between samples (ANOVA, Tukey, $\mathrm{P}<0.05$ ), and $\mathrm{n}=$ number of analyzed plants $(\mathbf{B})$ or samples (D).

Fig. 2. Two regions in LysM1 domains of NFR1 and CERK6 are required for chitin and Nod factor signaling. (A) Schematics of NFR1 and CERK6. Boxes represent LysM domains 
(TJ) and the kinase domain (KD). Dotted lines and amino acids specify boundaries for chimera design or mutations. (B/F) Nodules formed on $n f r l$ expressing indicated chimeras. (C/G) ROS production observed for wild type (WT) and cerk6 expressing indicated chimeras. In B, C, F and $\mathbf{G}$ letters indicate significant differences (ANOVA, Tukey, $\mathrm{P}<0.05$ ), and $\mathrm{n}=$ number of analyzed plants $(\mathbf{B} / \mathbf{F})$ or samples $(\mathbf{C} / \mathbf{G})$. (D) Model of the predicted chitin $(\mathrm{CO})$ binding groove in LysM1 and LysM2 of CERK6. Arrows indicate the location of the tryptophan (W) that was tested in chimeras (31) and (32). (E) Alignment of the LysM1 from NFR1 and CERK6. Identical amino acids are in grey. Regions I, II, III, and IV, beta strands $(\beta 1, \beta 2)$, alpha helices $(\alpha 1, \alpha 2)$ based on the CERK6 structure are indicated.

Fig. 3. Two regions in LysM1 domains of NFR1 and LYK3 are required for specificity of Nod factor signaling. (A) Alignment of the LysM1 domains of NFR1 and LYK3. Identical amino acids are in grey. Regions II, III, and IV are marked. Beta strands $(\beta 1, \beta 2)$, alpha helices $(\alpha 1, \alpha 2)$ based on the LYK3 structure are indicated (B) Schematic showing NFR1 and LYK3. Dotted lines and amino acids specify boundaries for chimera design. Boxes represent the ectodomain (EC) LysM domains (LysM1, LysM2 and LysM3), transmembrane + juxtamembrane domain (TJ) and the kinase domain (KD) (C) Chemical structures of $M$. loti and S. meliloti Nod factors. (D) Nodules formed on $n f r l$ expressing indicated chimeras (E) Nodules formed on lyk3 expressing indicated chimeras. In (D) and (E) letters indicate significant differences (ANOVA, Tukey, $\mathrm{P}<0.05$ ), and $\mathrm{n}=$ number of analyzed plants. Binding of Nod

20 factors to LYK3 (F), or NFR1 (G) ectodomains. Binding of M. loti Nod factor to CERK6 (H), or chimeric NFR1 with regions II and IV from CERK6 (I). In F to I binding of depicted (LYK3, CERK6 and NFR1) ectodomains (dilution series from 100, 50, 25, 12.5, 6.25, 3.13 and 1.56 $\mu \mathrm{M}$ ) to immobilized Nod factors. 
Fig. 4. Crystal structure of LYK3 reveals differences in Nod factor versus a chitin binding site. (A) Cartoon representation of the crystal structure of LYK3 ectodomain with LysM1, LysM2 and LysM3 colored as indicated. Glycosylations are in grey, the three conserved disulfide bridges in yellow. (B) Structural superposition of LYK3 with CERK6 (PDB: 5LS2 (21, 41)) in green. (C) Close-up of LysM1 showing the structural differences between LYK3 and CERK6. Superposition of chitotetraose from the AtCERK1 crystal structure (PDB: 4EBZ (프)) onto LysM1 from CERK6 (D) or LYK3 (E) with the identified regions II and IV highlighting the ligand binding site. No steric clashes are observed for CERK6 to the superpositioned ligand. Location of P87S mutation in $l y k 3$ and L77P in RisNod4 mutants are shown.

Fig. 5. Engineering of CERK6, NFR1 and LYK3 for specific Nod factor recognition. (A) Modelling conservation of NFR-type receptors onto the structure of LYK3 LysM1. Regions II, III and IV are highlighted in blue. B) Modelling conservation of CERK-type receptors onto the structure of CERK6 LysM1. Regions II and IV are highlighted in green. For (A) and (B) the thickness of the cartoon representation signifies conservation. The alignment logos of regions II, III and IV are shown in boxes. (C) Nodules formed on $n f r l$ expressing indicated chimeras. (D) Nodules formed on lyk3 expressing indicated chimeras. In (C) and (D) letters indicate significant differences (ANOVA, Tukey, $\mathrm{P}<0.05$ ) and $\mathrm{n}=$ number of analyzed plants. $\mathbf{E}$ ) Binding of the ectodomain of CERK6 (green) with regions II and IV from NFR1 (white) (dilution series from $12.5,6.25$ and $3.13 \mu \mathrm{M})$ to immobilized M. loti Nod factor. 
A

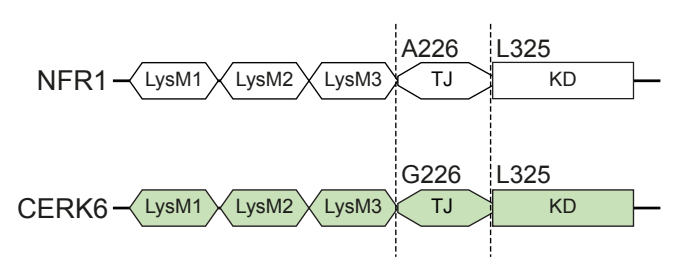

B

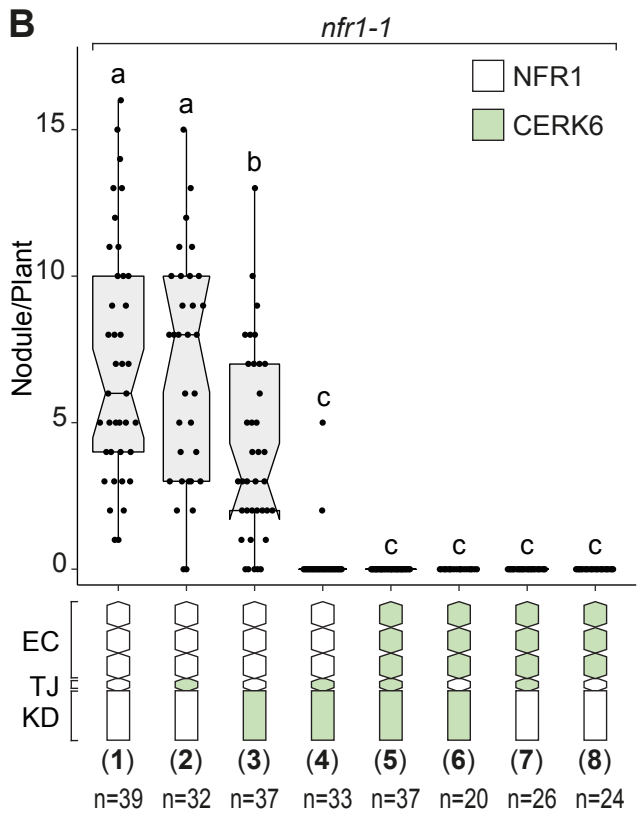

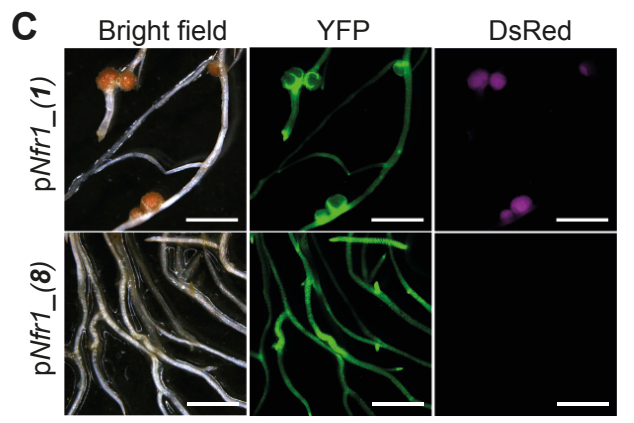

D $\quad$ WT cerk6-1
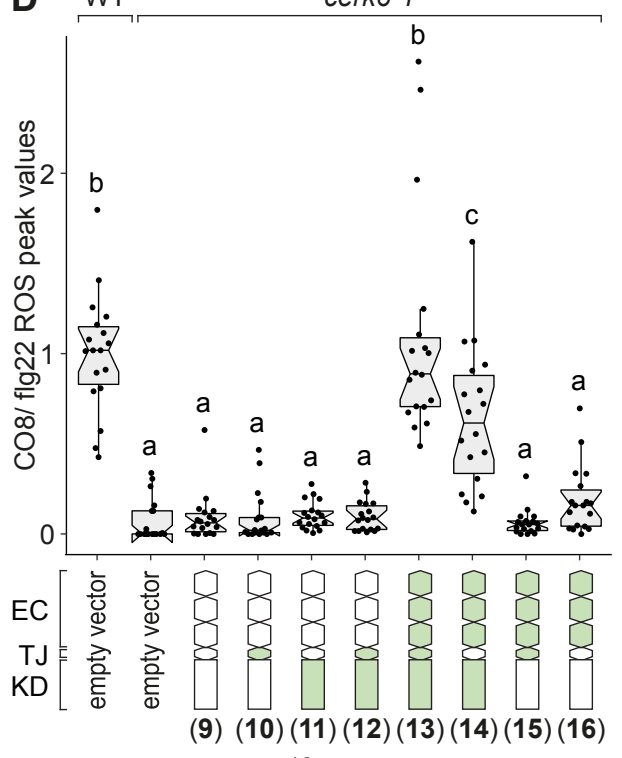

Fig. 1. Chimeric receptors reveal critical domains for activation of chitin and Nod factor signaling (A) Schematics of NFR1 and CERK6. Boxes represent the ectodomain (EC), LysM domains (LysM1, LysM2 and LysM3), transmembrane + juxtamembrane domain (TJ) and the kinase domain (KD). Dotted lines and amino acids specify boundaries. (B) Nodules formed on $n f r 1$ roots expressing indicated chimeras under control of the $N f r l$ promoter. $\mathrm{n}=$ number of analysed plants. (C) $n f r l$ roots expressing chimeras (1/8), scale bar $=5 \mathrm{~mm}$. (D) ROS production observed for wild type (WT) and cerk6 roots expressing indicated constructs under control of the Cerk6 promoter. $\mathrm{n}=$ number of individual biological samples. In $(\mathbf{B})$ and $(\mathbf{D})$ different letters indicate significant differences between samples (ANOVA, Tukey, $\mathrm{P}<0.05$ ) 
A

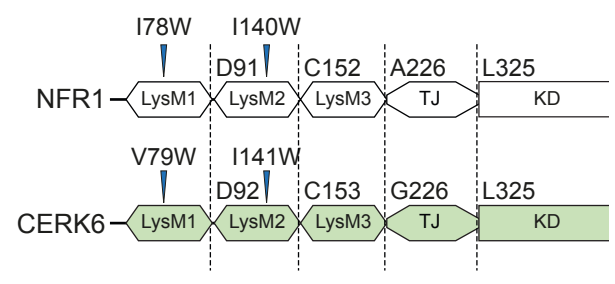

D

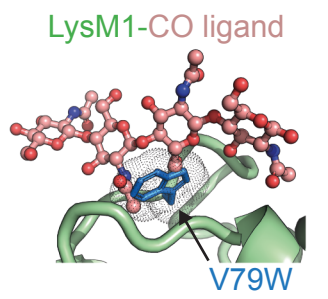

LysM2-CO ligand

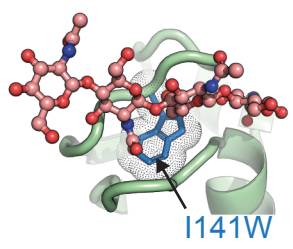

B
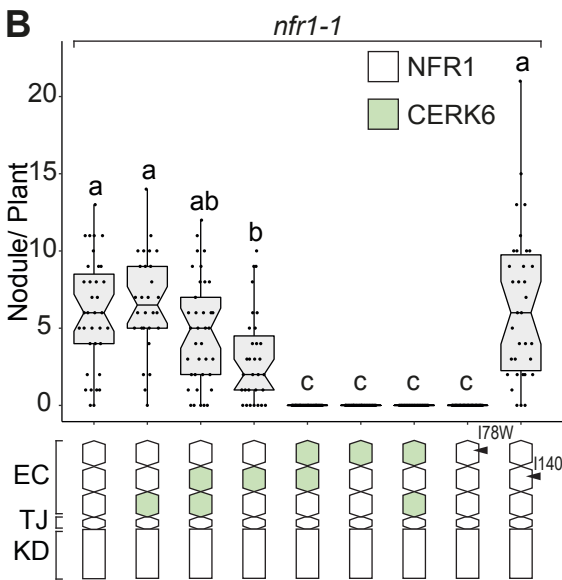

(1) $(17)(18)(19)(20)(21)(22)(23)(24)$ $n=39 n=28 n=37 n=31 n=36 n=34 n=30 n=39 n=34$
C WT cerk6-1

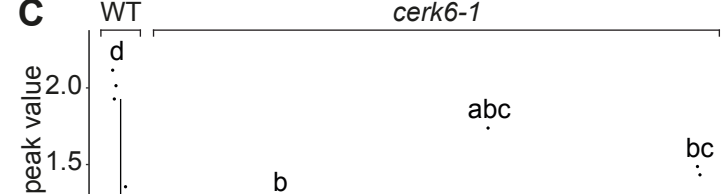

$\mathrm{bc}$

ac

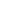

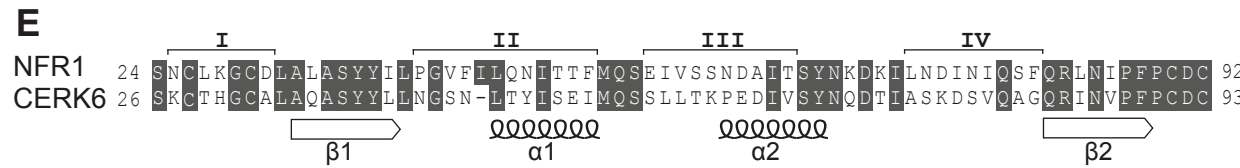

$\mathbf{F}$
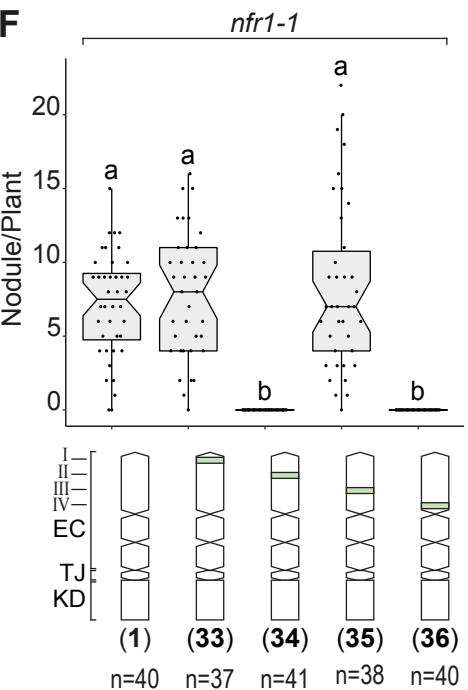
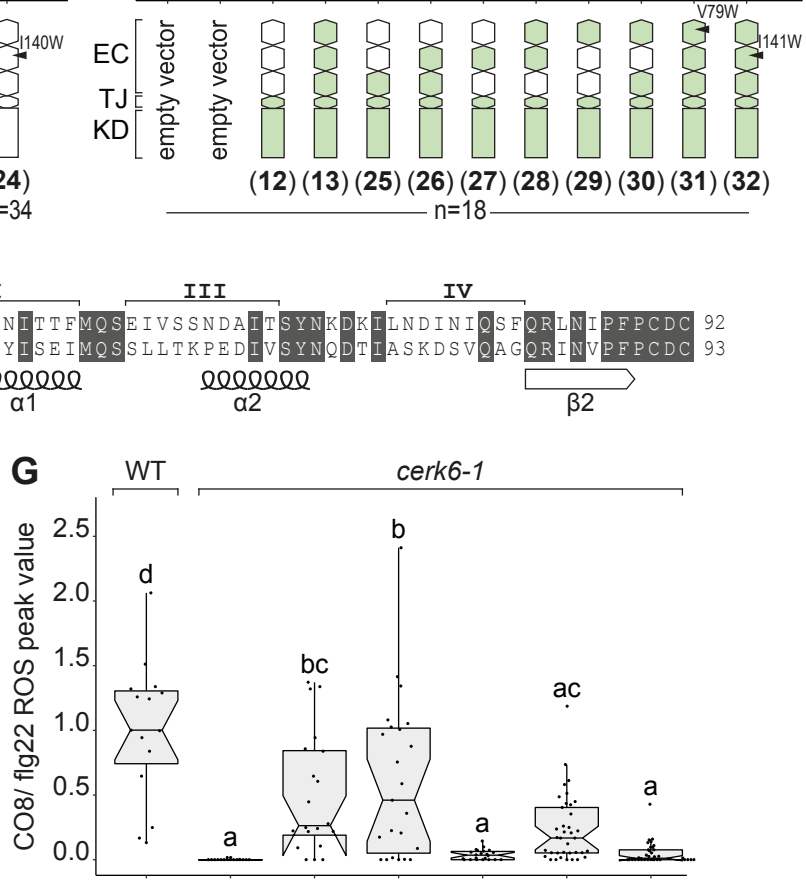

$(12)(13)(25)(26)(27)(28)(29)(30)(31)(32)$

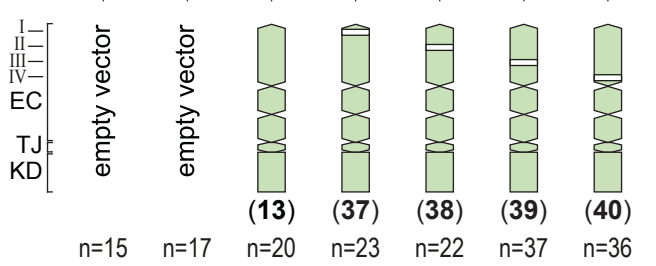

Fig. 2. Two regions in LysM1 domains of NFR1 and CERK6 are critical for chitin and Nod factor signaling. (A) Schematics of NFR1 and CERK6. Boxes represent the ectodomain (EC), 
the kinase domain (KD). Dotted lines and amino acids specify boundaries. (B/F) Nodules formed on $n f r l$ roots expressing indicated chimeras under control of the $N f r l$ promoter. $\mathrm{n}=$ number of analyzed plants. (C/G) ROS production observed for wild type (WT) and cerk6 roots expressing indicated constructs under control of the Cerk6 promoter. $\mathrm{n}=$ number of individual biological samples. In $\mathbf{B}, \mathbf{C}, \mathbf{F}$ and $\mathbf{G}$ different letters indicate significant differences between samples (ANOVA, Tukey, $\mathrm{P}<0.05$ ). (D) Model of the predicted chitin $(\mathrm{CO})$ binding groove in LysM1 and LysM2 of CERK6. Arrows indicate the location of the tryptophan (W) that was inserted to create chimeras (31) and (32). E) Alignment of the LysM1 domains of NFR1 and CERK6.

Identical amino acids are marked in grey. Regions I, II, III, and IV are marked. Beta sheets ( $\beta 1$, $\beta 2)$, alpha helices $(\alpha 1, \alpha 2)$ based on the CERK6 crystal structure are indicated. 


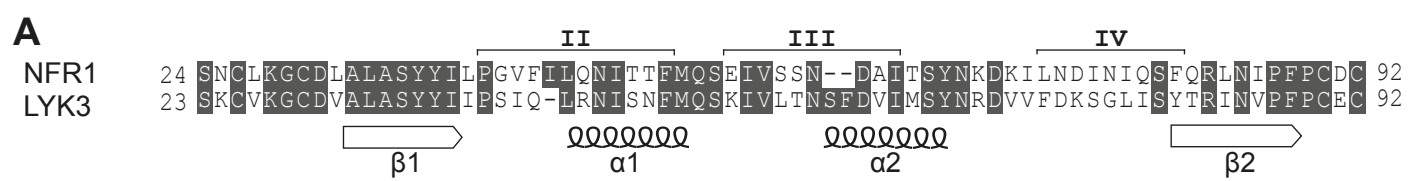

B

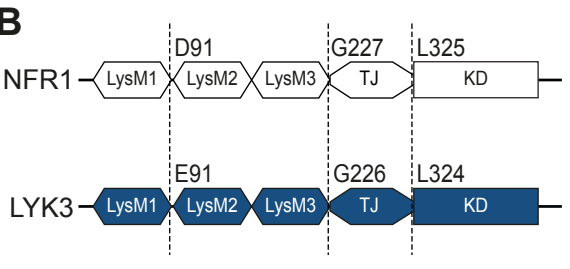

C
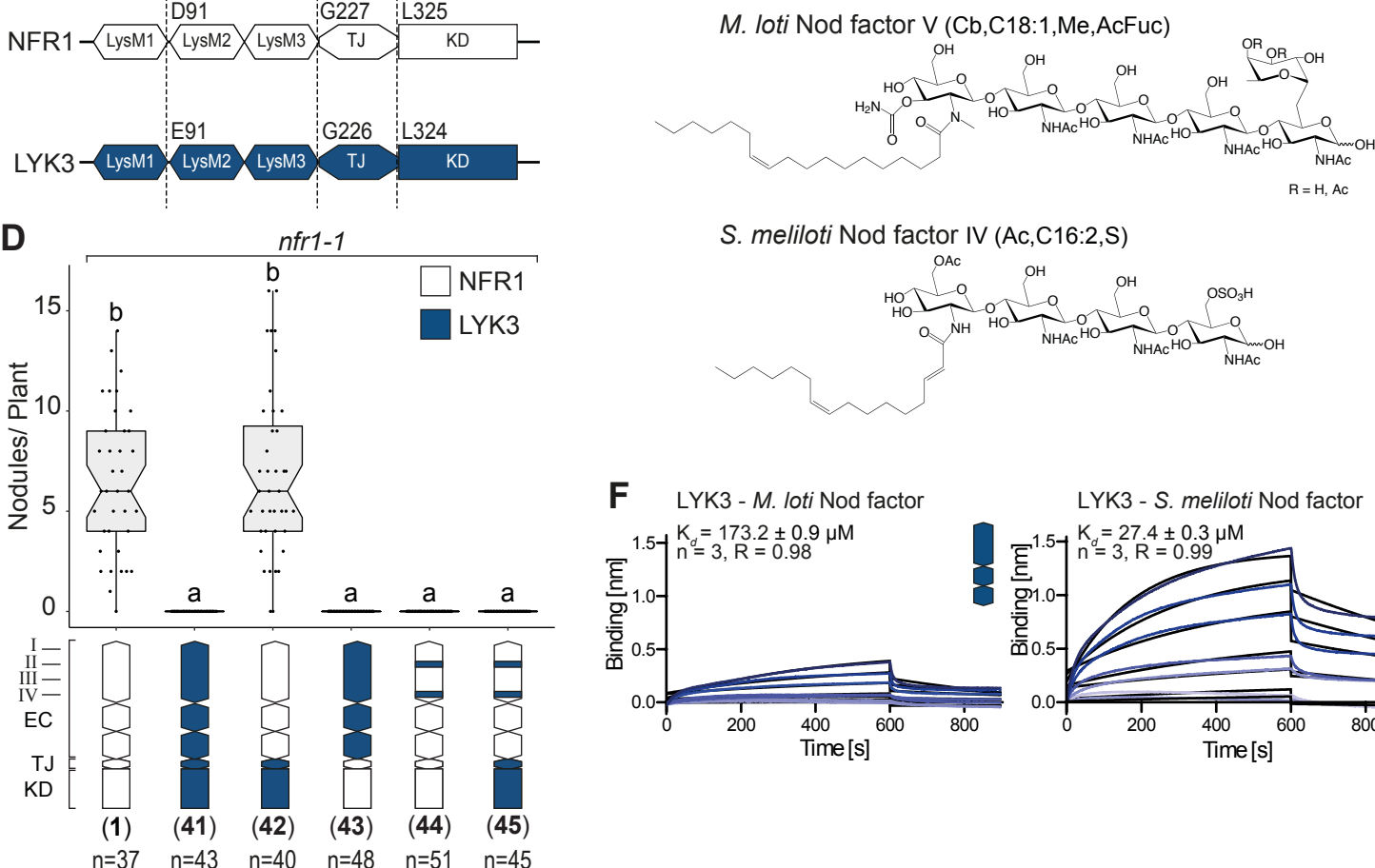

S. meliloti Nod factor IV (Ac,C16:2,S)
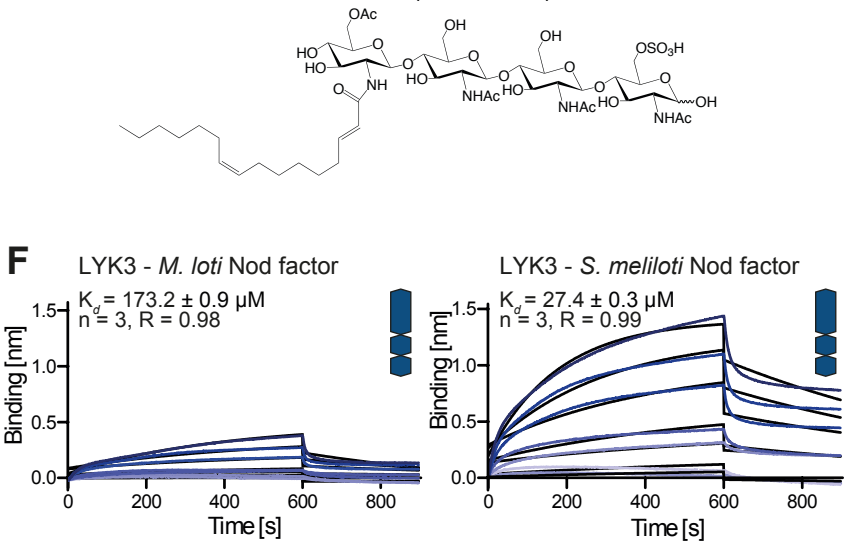

$n=37 \quad n=43 \quad n=40 \quad n=48 \quad n=51 \quad n=45$
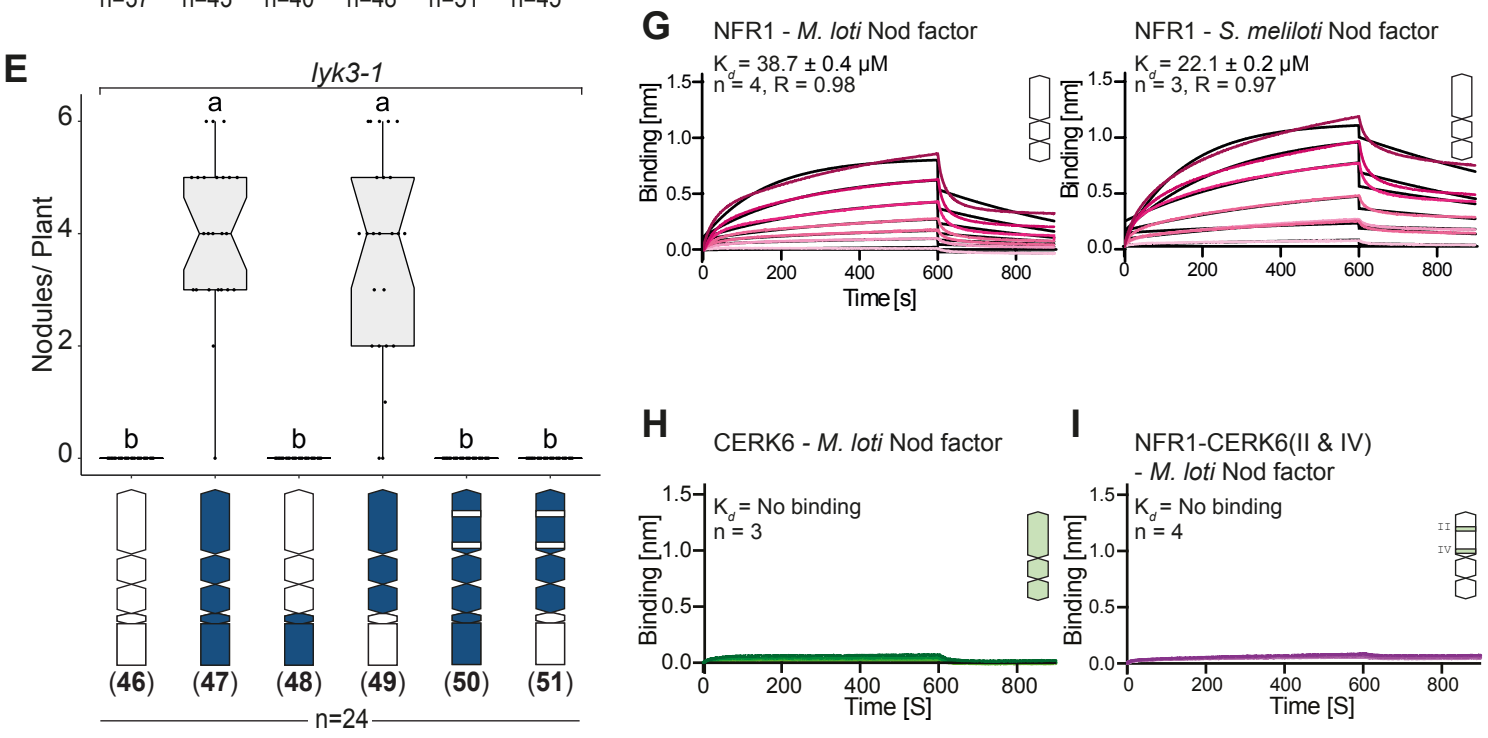

Fig. 3. Two regions in LysM1 domains of NFR1 and LYK3 are critical for specificity of Nod factor signaling. (A) Alignment of the LysM1 domains of NFR1 and LYK3. Identical amino acids are marked in grey. Regions II, III, and IV are marked. Beta sheets $(\beta 1, \beta 2)$, alpha helices $(\alpha 1, \alpha 2)$ based on the LYK3 crystal structure are indicated (B) Schematic showing NFR1 and LYK3. Dotted lines and amino acids specify boundaries for chimeric construct design. Boxes represent the ectodomain (EC), transmembrane + juxtamembrane domain (TJ) and the 
kinase domain (KD) (C) Chemical structures of M. loti and S. meliloti Nod factors. (D) Nodules formed on $n f r l$ roots expressing indicated chimeras under control of the $N f r l$ promoter. $\mathrm{n}=$ number of analyzed plants. (E) Nodules formed on $l y k 3$ roots expressing indicated chimeras under control of the $L y k 3$ promoter. $\mathrm{n}=$ number of analyzed plants. In (D) and (E) the different letters indicate significant difference among samples (ANOVA, Tukey, $\mathrm{P}<0.05$ ). (F) BLI measurement of Nod factor binding to ectodomains of LYK3 expressed and purified from insect cells. (G) BLI measurement of Nod factor binding to ectodomains of NFR1 expressed and purified from insect cells. (H) BLI measurement of M. loti Nod factor binding to ectodomains of CERK6 expressed and purified from insect cells. (I) BLI measurement of $M$. loti Nod factor binding to chimeric NFR1 ectodomains with regions II and IV from CERK6. 

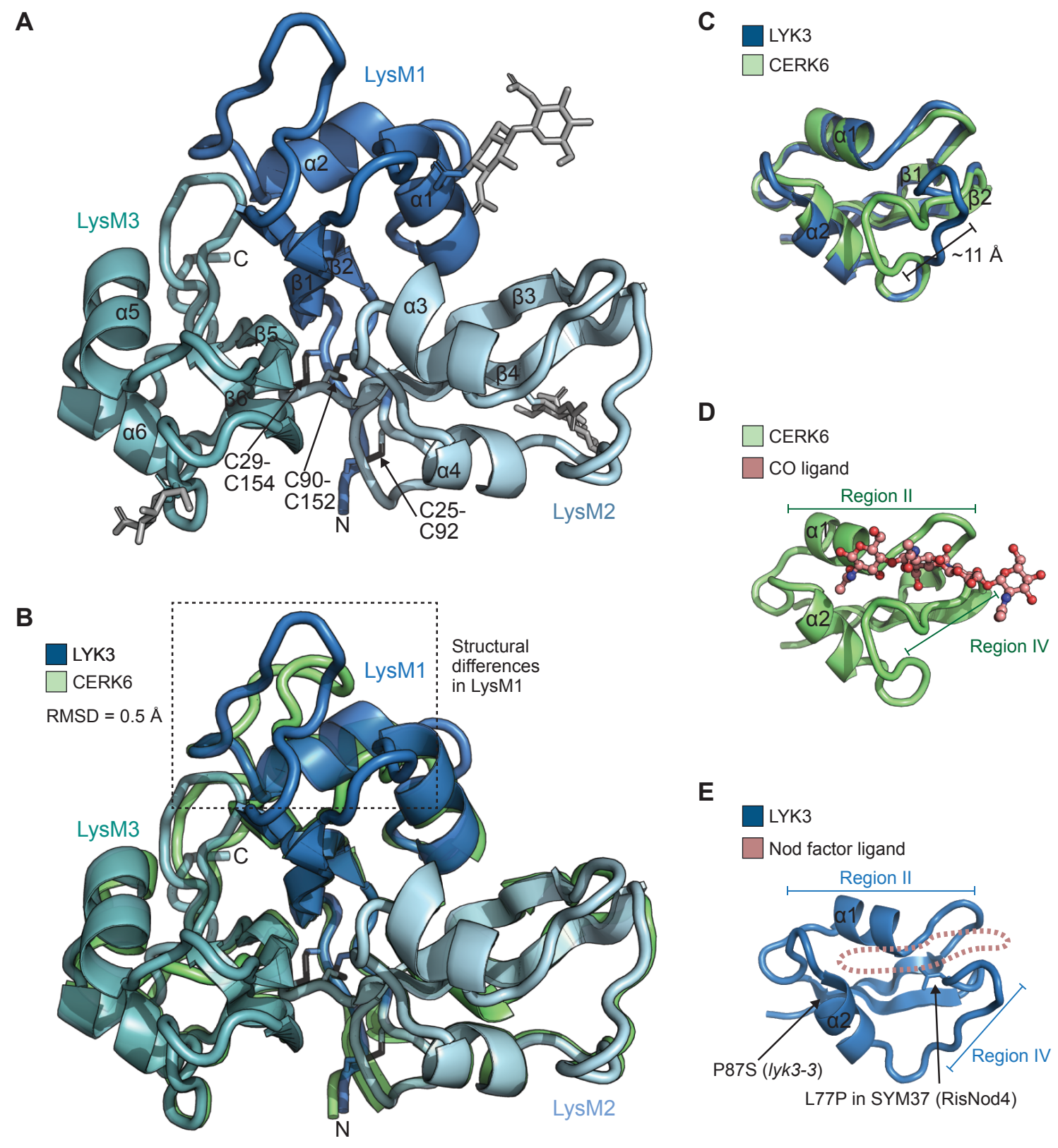

Fig. 4. Crystal structure of LYK3 reveals differences in a Nod factor versus a chitin binding site. (A) Cartoon representation of the crystal structure of Medicago LYK3 ectodomain with LysM1, LysM2 and LysM3 colored as indicated. Glycosylations are shown in grey, the three conserved disulfide bridges in black. (B) Structural superposition of LYK3 with the chitin receptor CERK6 (PDB: 5LS2) in green. (C) Close-up of LysM1 showing the structural differences between LYK3 and CERK6. Superposition of chitotetraose from the AtCERK1 crystal structure (PDB: 4EBZ) onto LysM1 from CERK6 (D) or LYK3 (E) with the identified regions II and IV highlighting the ligand binding site. No steric clashes are observed for CERK6 to the superpositioned CO ligand. Location of P87S mutation in lyk3-3 and L77P in RisNod4 mutants are shown. 

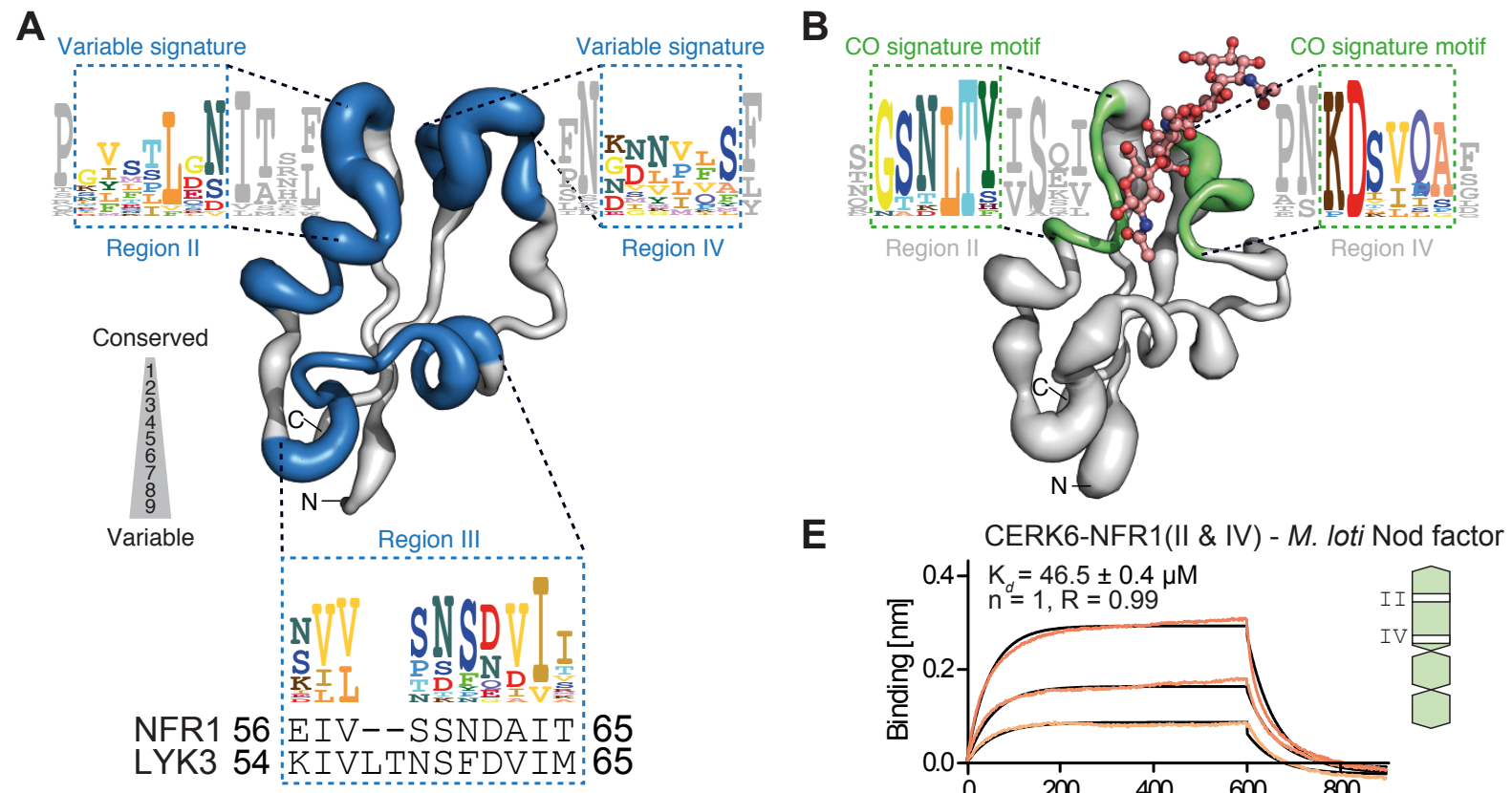

$\mathbf{E}$
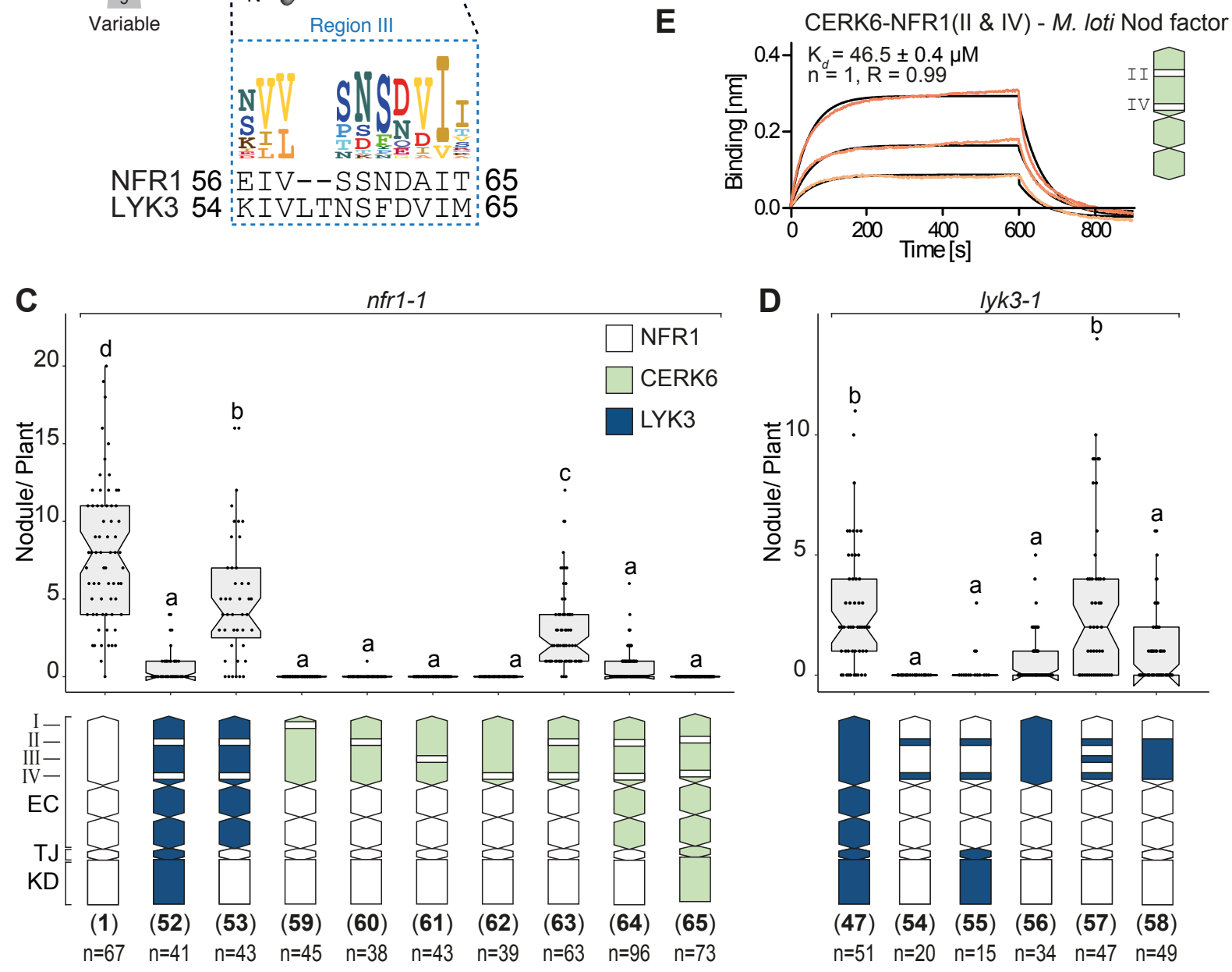

Fig. 5. Engineering of CERK6, NFR1 and LYK3 for specific Nod factor recognition. (A) Modelling conservation of NFR-type receptors onto the structure of LYK3 LysM1. Regions II, III and IV are highlighted in blue. The alignment logos of regions II, III and IV are shown in boxes. B) Modelling conservation of CERK-type receptors onto the structure of CERK6 LysM1. Regions II and IV are highlighted in green. For (A) and (B) the thickness of the backbone atoms (in grey) signifies conservation. The alignment logos of indicated regions are shown in boxes. (C) Nodules formed on $n f r 1$ roots expressing indicated chimeras under control of the $N f r 1$ promoter. $\mathrm{n}=$ number of analyzed plants. (D) Nodules formed on $l y k 3$ roots expressing indicated 
chimeras under control of the $L y k 3$ promoter. $\mathrm{n}=$ number of analyzed plants. In $(\mathbf{C})$ and (D) the different letters indicate significant difference among samples (ANOVA, Tukey, $\mathrm{P}<0.05$ ). E) BLI measurement of $M$. loti Nod factor binding to chimeric ectodomains of CERK6 with regions II and IV from NFR1 expressed and purified from insect cells. The scheme shows the composition of the ectodomain with parts of CERK6 in green and parts of NFR1 in white. 